\title{
DIELECTRIC PROPERTIES OF MATERIALS AT CRYOGENIC TEMPERATURES AND MICROWAVE FREQUENCIES
}

\author{
Richard G. Geyer \\ National Institute of Standards and Technology, 325 Broadway, Bouider, CO 80303 U.S.A. \\ Jerzy Krupha \\ Instytut Mikroelektroniki i Optoelektroniki Politechniki Warszawsliej, Koszykowa 75, 00-662 Warszawa, POLAND
}

\section{Abstract}

The permittivity and dielectric loss tangent of singlecrystal quartz, cross-linked polystyrene (Rexolite), and poly tetrafluoroethylene (Teflon) were measured at microwave frequencies and at temperatures of $77 \mathrm{~K}$ and $300 \mathrm{~K}$ using a dielectric resonator technique. Application of high-temperature superconducting (HTS) films as the endplates of the dielectric resonator made it possible to determine dielectric loss tangents of about $T$ $x 10^{-6}$ at $77 \mathrm{~K}$. Two permittivity tensor components for uniaxially anisotropic crystalline quartz were measured. Although the permittivities at $77 \mathrm{~K}$ changed very little from their room temperature values at $300 \mathrm{~K}$, large changes in dielectric losses were observed. The decreased loss characteristics of these microelectronic substrates can markedly improve the performance of many microwave devices at cryogenic temperatures.

\section{Introduction}

The performance parameters of many microwave devices depend on dissipation losses at the operating frequency and ambient temperature of the device. Often these performance parameters are limited by dielectric substrate losses. Many attempts have been made to characterize the dielectric properties of materials which are used for deposition of HTS films [1-3], which possess unique crystalline lattice structures that allow ad herence of the HTS film. The dielectric characteristics at cryogenic temperatures of other commonly used microelectronic substrates are also important in assessing potential performance gains of microwave devices operated at low temperature. In this paper a very sensitive method is used to measure permittivity and dielectric loss tantgent of cylindrical samples of dielectric substrates. This method makes it possible to measure dielectric loss tangents on the order of $10^{-6}$ accurately.

\section{Measurements}

The resonant system which was used to measure permittivity and dielectric loss tangent at cryogenic temperatures is shown in Fig. 1. The dielectric sample under test constitutes a cylindrical dielectric resonator situated between two parallel superconducting films ( $i$ i $(i)$

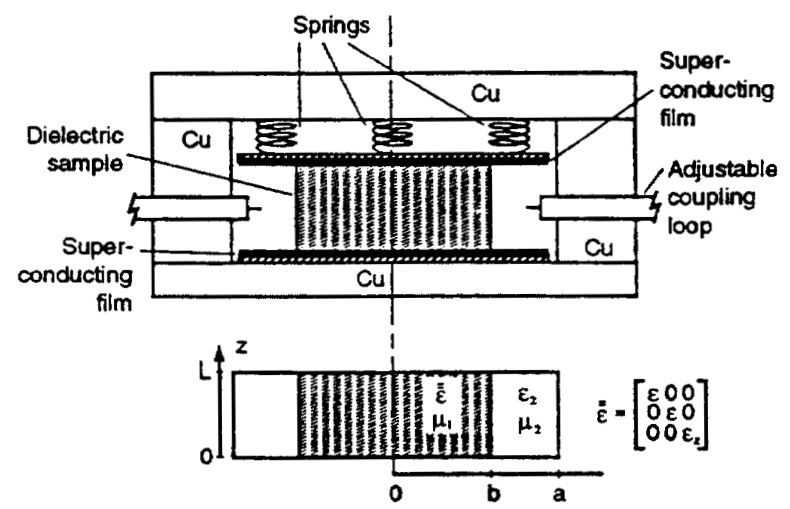

Figure 1: Resonant setup for measurement of permittivity $\overline{\bar{\epsilon}}$ and dielectric loss tangent at cryogenic temperatures.

or copper plates ( $300 \mathrm{~K}$ ). Provided that the sample faces are optically finished, there are no air gaps between the sample and the endplates. The cylindrical samples may. in general, possess uniaxial anisotropy along the cylinder axis.

Measurements on isotropic materials are performed using one of the $T E_{0 n !}$ modes (usually the $T E_{011}$ mode). The same circularly symmetric modes are also used for measurements of the permittivity tensor component $(\epsilon)$ that is perpendicular to the anisotropy axis of uniaxially anisotropic substrates. For anisotropic materials, determination of the parallel permittivity tensor component $\left(\epsilon_{z}\right)$ requires additional experiments with one of the hy. brid or TM modes. We have used the $H E_{111}$ and the $\mathrm{HE}_{211}$ modes since they can be easily excited. The permittivity perpendicular to the anisotropy axis has been evaluated by solving the characteristic equation for the $\mathrm{TE}_{\mathrm{On}}$ ! mode family, while taking into account the geometric dimensions of the resonant structure and the resonant frequency for the appropriate mode. Since we know $\epsilon$ and the resonant frequency for at least one hybrid mode, we can evaluate $\epsilon_{z}$ by solving the following hybrid mode characteristic equation, valid for $k_{2} \geq 0$,

$$
\left[\frac{\sqrt{\epsilon \epsilon_{z}} J_{m}^{\prime}\left(k_{\rho} b\right) X_{m}\left(k_{2} b\right)}{k_{1} J_{m}\left(k_{\rho} b\right) Q_{m}\left(k_{2} b\right)}+\frac{c_{2} X_{m}^{\prime}\left(k_{2} b\right)}{k_{2} Q_{m}\left(k_{2} b\right)}\right] \times
$$




$$
\begin{array}{r}
{\left[\frac{\mu_{1} J_{m}^{\prime}\left(k_{1} b\right) Q_{m}\left(k_{2} b\right)}{k_{1} J_{m}\left(k_{1} b\right) X_{m}\left(k_{2} b\right)}+\frac{\mu_{2} Q_{m}^{\prime}\left(k_{2} b\right)}{k_{2} X_{m}\left(k_{2} b\right)}\right]} \\
=\frac{m^{2} c^{2} k_{2}^{2}}{\omega^{2} b^{2}}\left[\frac{1}{k_{1}^{2}}+\frac{1}{k_{2}^{2}}\right]^{2},
\end{array}
$$

where

$$
\begin{aligned}
& X_{m}\left(k_{2} \rho\right)=K_{m}\left(k_{2} \rho\right)-\frac{K_{m}\left(k_{2} a\right)}{I_{m}\left(k_{2} a\right)} I_{m}\left(k_{2} \rho\right), \\
& Q_{m}\left(k_{2} \rho\right)=K_{m}\left(k_{2} \rho\right)-\frac{K_{m}^{\prime}\left(k_{2} a\right)}{I_{m}^{\prime}\left(k_{2} a\right)} I_{m}\left(k_{2} \rho\right), \\
& X_{m}^{\prime}\left(k_{2} \rho\right)=K_{m}^{\prime}\left(k_{2} \rho\right)-\frac{K_{m}\left(k_{2} a\right)}{I_{m}\left(k_{2} a\right)} I_{m}^{\prime}\left(k_{2} \rho\right), \\
& Q_{m}^{\prime}\left(k_{2} \rho\right)=K_{m}^{\prime}\left(k_{2} \rho\right)-\frac{K_{m}^{\prime}\left(k_{2} a\right)}{I_{m}^{\prime}\left(k_{2} a\right)} I_{m}^{\prime}\left(k_{2} \rho\right), \\
& k_{1}^{2}=\frac{\omega^{2}}{c^{2}} \epsilon \mu_{1}-k_{z}^{2}, \quad k_{2}^{2}=k_{z}^{2}-\frac{\omega^{2}}{c^{2}} \epsilon_{2} \mu_{2}, \\
& k_{\rho}^{2}=k_{1}^{2} \frac{\epsilon_{z}}{\epsilon}, \quad k_{z}=\frac{I_{1}}{L},
\end{aligned}
$$

and $J_{m}$ is a Bessel function of first kind, $I_{m}, K_{m}$ are modified Bessel functions, $\omega$ is angular frequency, $c$ is the speed of light, $b, a$ are the radii of sample and cylindrical cavity, $L$ is sample height, $l$ is an integer $1,2,3, \ldots$ denoting number of half-wavelength variations along zdirection, and ' represents differentiation. The dielectric loss tangent for $T E_{011}$ mode excitation is evaluated from

$$
\tan \delta=p_{e}\left(1 / Q_{u}-1 / Q_{c}\right),
$$

where $Q_{u}$ is the unloaded $Q$ factor for the resonant system with dielectric sample, $Q_{c}$ is the $Q$ factor depending on conductor losses, and $p_{e}$ is the electric-energy filling factor, which can be computed from the permittivity of the dielectric sample under test. Evaluation of $Q_{c}$ requires measurements of the surface resistances of the HTS films used and of copper. For measurements at $77 \mathrm{~K}, 76 \mathrm{~mm}$ diameter yttrium- barium-copper-oxide (YBCO) films deposited on buffered sapphire substrates were used.

A variational uncertainty analysis which includes sources of measurement error due to dielectric sample dimensions, surface resistance of resonator endplates, and resonance frequency and $Q$ factor determination was performed. Systematic errors in the determination of $c$ are only weakly affected by imperfections of the dielectric sample faces, since for TE modes the electric field has only an azimuthal component tangential and contiguous with the sample surface. Measurement uncertainties for $\epsilon_{z}$, however, are strongly affected by endplate air-gap effects. These uncertainties increase as $\epsilon_{z}$ increases and as the dielectric sample aspect ratio (2b/L) decreases. Typical total combined uncertainties for wellmachined samples are 0.3 percent for $\epsilon$ and $1-2$ percent for $\epsilon_{z}$ (if $\epsilon_{z}$ does not exceed 30-40).

The results of laboratory experiments as a function of temperature $T$ and frequency $f$ are summarized in $T a-$ ble 1. The quartz crystal sample was c-axis oriented,
Table 1: Dielectric properties of crystalline quartz, Rexolite, and Teflon at cryogenic temperatures and microwave frequencies.

\begin{tabular}{|c|c|c|c|c|}
\hline Material & $\epsilon$ & $\tan \delta$ & $\mathrm{T}(\mathrm{K})$ & $\mathrm{f}(\mathrm{GHz})$ \\
\hline Quartz & $4.443^{A}$ & $1.3 \times 10^{-5}$ & 300 & 9.03 \\
& $4.590^{B}$ & - & 300 & 7.75 \\
& $4.424^{A}$ & $7.0 \times 10^{-6}$ & 77 & 9.06 \\
& $4.580^{B}$ & -- & 77 & 7.77 \\
\hline Rexolite & 2.558 & $4.0 \times 10^{-4}$ & 300 & 13.0 \\
& 2.504 & $2.5 \times 10^{-4}$ & 77 & 13.0 \\
\hline Teflon & 2.055 & $2.0 \times 10^{-4}$ & 300 & 9.93 \\
& 2.048 & $8.0 \times 10^{-6}$ & 77 & 9.95 \\
\hline
\end{tabular}

${ }^{A}$ Normal to c-axis. ${ }^{B}$ Parallel to c-axis.

and loss tangent results are shown only for the $T E_{011}$ mode measurements taken perpendicular to the axis of anisotropy. The room-temperature measurements compare well with those previously reported [4]

\section{Summary}

Use of the sample under test as a dynamic dielectric rod resonator and HTS films as endplates of the resonator allowed accurate dielectric substrate measurements to be made at cryogenic temperatures. With proper identification of excited hybrid modes, the tensor permittivity components of uniaxially anisotropic materials may be determined. Generally, permittivities of the tested dielectric substrates at cryogenic temperatures varied little from their room-temperature values. However, changes in dielectric loss characteristics at cryogenic temperatures can be significant. When dielectric losses in substrates are important in microwave device application, they should be measured and specified at the operating temperature and frequency.

\section{$5 \quad$ References}

1. W.G. Lyons, "Low-loss substrates for hightemperature superconductors," MIT Lincoln Lab. Quart. Rep., October, 1989.

2. J. Konopka and I. Wolff. "Dielectric properties of substrates for deposition of high- $T c$ thin films up to $40 \mathrm{GHz}$." IEEE Trans. Microwave Theory Tech. vol. 40. pp. 2418-24:3, 1993.

3. J. Krupka, R.G. Geyer, M. Kuhn, and J. Hinken, "Dielectric properties of single crystals of $\mathrm{Al}_{2} \mathrm{O}_{3}$. $\mathrm{LaAlO}_{3}, \mathrm{NdGaO}_{3}, \mathrm{SrTiO}_{3}$, and $\mathrm{MgO}$ at cryogenic temperatures," IEEE Trans. Microwave Theory Tech. (to be published), 1994.

4. J.R. Birch et al., "An intercomparison of measurement techniques for the determination of the dielectric properties of solids at near millimetre wavelengths," National Phys. Lab. Rep. DES 115, October, 1991 .

U.S. Government work not protected by U.S. copyright 